\title{
Total normal
}

\section{Kinder mit Behinderung in Tageseinrichtungen - Beispiel: die Rahmenvereinbarung Integrationsplatz in Hessen}

Marek Körner

Marek Körner ist Referent Soziale Arbeit des Paritätischen Wohlfahrtsverbandes, Landesverband Hessen e. V. und Geschäftsführer der Paritätischen Projekte gGmbH.

E-Mail

Marek.Koerner@paritaethessen.org
Integration lebt von der Idee einer gemeinsamen Erziehung von Kindern mit und ohne Behinderung. Diese Idee fordert Träger, Organisation und insbesondere Fachkräfte heraus, pädagogische Haltungen und Lösungen zu entwickeln, die ausnabmslos alle Kinder einer Kindergruppe gleichermaßen und gemäß ibrer spezifischen Bedürfnissen, betreut, erzieht und bildet. Wie dieser Anspruch in der Praxis ausgestaltet wird, kann das Beispiel des Bundeslandes Hessen zeigen.

In der bundesdeutschen fachlichen und sozialpolitischen Diskussion der letzten Jahrzehnte wurden die zentralisierten Betreuungs und Unterbringungsformen in der Behindertenhilfe, institutionalisierter Ausdruck tradierter preußischer ordnungs und sozialpolitischer Strukturen, zunehmend in Frage gestellt. Die Träger von Einrichtungen reflektierten ihre institutionelle Arbeit immer weniger an den Aspekten der gesellschaftlichen Legitimation behinderte Menschen in spezialisierten (Sonder) Einrichtungen zu betreuen, sondern an ihrem Anspruch, individuell unterstützende Arrangements zu konzeptionieren, die an einem konkreten Hilfebedarf der zu betreuenden Menschen orientiert waren. Die daraus resultierenden Arbeitskonzepte und Handlungsansätze der Betreuungsarbeit mussten sich auf Grund individueller Bedarfe zwangsläufig an wechselnden Ansprüchen und Wünschen der Menschen orientieren und sich zunehmend durch Offenheit, Flexibilität und Gestaltbarkeit des organisatorischen Rahmens auszeichnen.

Nachhaltig beeinflussende Aspekte in der fachlichen und methodischen Diskussion der Behindertenhilfe waren die seit den 1960er Jahren einsetzenden gesellschaftlichen Prozesse der Differenzierung und Individualisierung, sowie die Anfang der 1990er Jahre beginnende, sozialpolitische und rechtliche Umorientierungen, die in der Folgezeit eine weitgreifende Emanzipation aus dem psychiatrischen
Ansatz ermöglichte. Die bis dahin zuverlässig (miss) verstandene Integration behinderter Menschen im Rahmen von Rehabilitation, als einseitige Anpassung des behinderten Menschen an geltende soziale Normen und Kompensation der individuellen Defizite und Beeinträchtigungen, wurde durch neue, insbesondere habilitationsorientierte Leitbegriffe ersetzt: Normalisierung, Inklusion, Integration und Selbstbestimmung waren Ausdruck des Anspruches die Integration der Menschen mit Behinderung in einen gesamtgesellschaftlichen Lernprozess einzubeten und als ethisches und auf Grundrechten des Menschen beruhendes Ziel zu beziehen.

Die Ergänzung des Artikel 3 Absatz 3 Grundgesetz um den Satz: "Niemand darf wegen seiner Behinderung benachteiligt werden " sowie die Einführung des Sozialgesetzbuches IX - Rehabilitation und Teilhabe behinderter Menschen im Jahre 2001, trug diesem fachlichen Ansinnen explizit Rechnung.

Die Bundesarbeitsgemeinschaft der Landesjugendämter hatte bereits im April 1987 ihren Mitgliedern empfohlen »sich für die gemeinsame Erziehung von behinderten und nicht behinderten Kindern einzusetzen und die entsprechenden Rahmenbedingungen an Hand der vorgelegten Grundsätze in ihren Zuständigkeitsbereich zu schaffen« (aus: Empfehlung der BAGLJÄ, 91. Arbeitstagung). Nach diesen Grundsätzen ist Integration vorrangig eine Frage des pädagogischen Bewusstseins und erst danach eine Frage der Rahmenbedingungen. Als Grenzen der Integration werden nicht die Art oder die Schwere der Behinderung definiert, sondern die strukturellen Rahmenbedingungen unter denen sich Integration vollzieht. »Es darf keine Randgruppe von Kindern mit schwersten Behinderungen zurükkbleiben bzw. auf reine Sonderkindergärten verwiesen werden. « (ebenda)

Nach dem 1991 in Kraft getretenen Kinder und Jugendhilfegesetz hat nach $\mathbb{S}$ 1 Abs. 1 SGB VIII jeder junge Mensch ein 
Die »Rahmenvereinbarung Integrationsplatz« in Hessen

Im Jahre 1999 wurde in Hessen die »Rahmenvereinbarung Integrationsplatz « zwischen den kommunalen Spitzenverbänden sowie der Liga der Freien Wohlfahrtspflege geschlossen. Das Hessische Sozialministerium hat durch eine ergänzende Vereinbarung seine finanzielle Förderung daran rechtsverbindlich erklärt.

- Intention: Nach dem Grundgedanken der Rahmenvereinbarung soll jedes Kind mit Behinderung einen Integrationsplatz in einer wohnortnahen und seinen individuellen Bedürfnissen entsprechenden Tageseinrichtung erhalten. Damit soll jedem Kind mit Behinderung die Eingliederung in die Gesellschaft nach $\mathbb{S}$

53 ff SGB XII ermöglicht, sowie dem Anspruch des SGB VIII nach $\ 1$ Abs. 3 in einem Beitrag zur Förderung der individuellen und sozialen Entwicklung und der Vermeidung bzw. dem Abbau von Benachteiligung, entsprochen werden.

- Verknüpfung der Leistungsansprüche: Die organisatorische, finanzielle und vertragliche Verknüpfung der Leistungsansprüche des SGB VIII und SGB XII sind grundlegender Ausdruck der Rahmenvereinbarung Integrationsplatz. Die integrative Erziehung von Kindern mit Behinderung muss danach sowohl dem Erziehungs, Bildungs und Betreuungsauftrag der Kindertageseinrichtung nach $\ 22$ ff SGB VIII entsprechen als auch dem Eingliede-

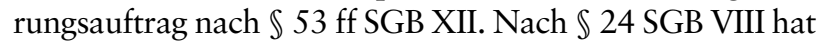
jedes Kind vom vollendeten dritten Lebensjahr bis zum Schuleintritt Anspruch auf den Besuch einer Tageseinrichtung. Ergänzend zu den elementarpädagogischen Leistungen der Kindertageseinrichtung werden einem Kind mit Behinderung aufgrund des Leistungsanspruchs nach $\mathbb{5} 53 \mathrm{Abs}$. 3 SGB XII zusätzliche Hilfen und Leistungen im Rahmen der Eingliederungshilfe durch die Einrichtung zur Verfügung gestellt. Diese Leistungen sind in der Rahmenvereinbarung beschrieben worden und umfassen $u$. a.:

$\circ$ individuelle und gruppenbezogene Entwicklungsbegleitung in integrativen Prozessen - Leben und Lernen in der Gruppe

- Erwerb von Grundfähigkeiten

- Erlernen von lebenspraktischen Fähigkeiten

- Förderung der ganzheitlichen Entwicklung von sprachlichen, motorischen, emotionalen und kognitiven Fähigkeiten

- Nahrungsaufnahme und Körperpflege

- Herstellen von Kontakten zwischen den Familien der Tageseinrichtung

- Begleitung der Familien

- Sicherstellung der heilpädagogischen Maßnahmen und Einbindung von allgemeiner und medizinischer Pflege bzw. Therapie in den Tagesablauf

- Zusammenarbeit mit Schulen

- Hilfeplanung, Dokumentation, Qualitätsentwicklung

- Fortbildung der Fachkräfte
Mit dieser Beschreibung werden Leistungsaspekte der therapeutischen und heilpädagogischen Betreuung und Versorgung in der Rahmenvereinbarung benannt und für eine gelingende Integrationspraxis auch als notwendig beschrieben. Die tatsächliche Leistungserbringung in der Kindertageseinrichtung ist laut Rahmenvereinbarung dann nur in einer organisatorischen und konzeptionellen Verknüpfung und gegenseitigen Durchdringung von Jugendhilfeleistungen nach dem Kinder und Jugendhilfegesetz und Sozialhilfeleistung nach der Eingliederungshilfe des SGB XII durchführbar.

Als Problem hat sich die eigenwillige Konstruktion der Zeit und Entgeltpauschale dargestellt. Nicht intendiert war die abgegrenzte Zuordnung der 15 Stunden ausschließlich auf Eingliederungsleistungen, was in der Praxis tatsächlich nur zu einer Betreuung von maximal drei Stunden am Tag führen würde. Viele kommunale Einrichtungen haben diese methodisch eingeschränkte Umsetzung aus Kostengründen praktiziert und den Ärger der Eltern und anderer Träger provoziert.

Auch die pauschale Definition des Entgeltsatzes ohne Anknüpfung an die Feststellung des individuellen Hilfebedarfes hat zu Problemen in der Aufnahme von schwerst und mehrfachbehinderten Kindern in Regeleinrichtungen geführt, da sich die Träger stellenweise nicht in der Lage sahen, die notwendigen Leistungen fachlich und strukturell umzusetzen. Die Forderung der Liga der Freien Wohlfahrtspflege zum Aufbau von sogenannten Schwerpunkteinrichtungen mit spezifischen Leistungsangeboten in den jeweiligen Kommunen, welche dann auch umfänglichere Bedarfe abdecken könnten, konnte nicht umgesetzt werden.

Der Wegfall der generellen Fahrtkostenübernahme sowie die Erhebung von Elternbeiträgen führte bei Familien in prekären finanziellen Lagen häufig zu einem Abbruch der Integration. Die individuelle Antragstellung der Eltern und die lang dauernden oder späten Bewilligungszusagen der Kostenträger haben viele Eltern verunsichert und nicht zu einer verbesserten Planungssicherheit insbesondere von berufstätigen Eltern geführt.

Marek Körner 
Recht auf Förderung seiner Entwicklung und auf Erziehung zu einer eigenverantwortlichen und gemeinschaftsfähigen Persönlichkeit. Jugendhilfe soll zur Verwirklichung dieses Rechts junge Menschen in ihrer individuellen und sozialen Entwicklung fördern und dazu beitragen, Benachteiligungen zu vermeiden oder abzubauen ( $\mathbb{1} 1$ Abs. 1 u. Abs. 3 SGB VIII) Die Jugendministerkonferenz betont danach stringent und schlüssig, dass die integrative Erziehung den Zielen des Erziehungs, Bildungs und Betreuungsauftrages der Kindertageseinrichtungen wie auch dem Eingliederungsauftrag gleichermaßen entsprechen muss und kann. (JMK 6./7. Juni 2002, Osnabrück) richtet. Zudem sollte es darum gehen, ein gemeinsames Bewusstsein der jeweiligen Akteure und Fachkräfte der unterschiedlichsten Handlungsfelder und Qualifikationen herzustellen.

\section{Beispiel Hessen: vom Sonderkindergarten zum Integrationsplatz}

In Hessen existierten verschiedene Formen der gemeinsamen Förderung von Kindern mit und ohne Behinderung in Kindertageseinrichtungen, die sowohl die Möglichkeit der Betreuung als »Einzelintegration« als auch in »integrativen

\section{"Die Grenzen der Integration liegen nicht in der Bebinderung sondern in den Rabmenbedingungen "}

Mit den rechtlichen und fachlichen Diskursen konstituierte sich Anfang 2000 immer deutlicher der pädagogische Anspruch, eine Gesamtverantwortung für eine »Bildung für alle Kinder « gemeinsam umzusetzen. Dabei sollte der Anspruch auf Chancengerechtigkeit, Teilhabe und ein partizipatives Bildungsverständnis der Akteure handlungsleitend sein. Die Bedeutung von Bildung als offene Gemeinschaftsaufgabe ergibt sich vor dem Hintergrund, alle Kinder vor den Herausforderungen der Zukunft entsprechend $\mathrm{zu}$ qualifizieren, herkunftsbedingte ungleiche Ausgangsbedingungen auszugleichen und die junge Generation zu befähigen, dass sie am gesellschaftlichen Geschehen möglichst eigenständig teilnehmen und verantwortlich mitwirken kann.

Mit den fachlichen Neuorientierungen und den rechtlichen Veränderungen ging das beschriebene, sich in Veränderung befindliche Institutionenverständnis der Einrichtungsträger einher. Durch die Verknüpfung der Jugendhilfeleistungen des SGB VIII mit den Eingliederungsleistungen des SGB XII (ehemals BSHG) wurden umfassende Ansprüche an die Reorganisation von Arbeitsstrukturen, Verfahren, Routinen und Standards ge-
Gruppen« eröffnete. Die Form der Betreuung von Kindern mit Behinderungen in eigens dafür geschaffenen Einrichtungen wurde Ende 1998 von der Förderung seitens der Hessischen Landesregierung ausgenommen und der letzte »Sonderkindergarten « wurde 1999 geschlossen.

Bereits vor 1998 wurden jedoch auch in Hessen verschiedene Formen der gemeinsamen Förderung von Kindern mit Behinderung in Kindertageseinrichtungen umgesetzt. Neben der Finanzierung der »integrativen Gruppen« durch den Landeswohlfahrtsverband Hessen (LWV) als überörtlichen Träger, bestand bereits seit 1996 die »Rahmenvereinbarung Einzelintegration ", die es dem örtlichen Sozialhilfeträger ermöglichte, Einzelintegration in Regeltageseinrichtungen umzusetzen. Im Rahmen der außerordentlichen quantitativen Zunahme der integrativen Betreuungsmaßnahmen kam es laut dem Landeswohlfahrtsverband Hessen jedoch nachfolgend in der Umsetzung zu großen finanziellen und administrativen Problemen, insbesondere verschärft durch die gesplittete Sach und Kostenzuständigkeit bzw. durch das Delegationsverfahren und dessen negative verwaltungsbezogenen Auswirkung auf die Sozialhilfeträger.
Zum 31. Juli 1999 wurde die »Rahmenvereinbarung Einzelintegration « durch die Kostenträger Landeswohlfahrtsverband Hessen, Hessischer Städtetag und Hessischer Landkreistag gekündigt, um die integrativen Fördermaßnahmen in einer neuen Rahmenvereinbarung zusammenzuführen (»Angebote für Kinder mit Behinderung vom vollendeten 3. Lebensjahr bis Schuleintritt in Tageseinrichtungen für Kinder - Rahmenvereinbarung Integrationsplatz « - vgl. Kasten »Die $>$ Rahmenvereinbarung Integrationsplatz in Hessen «). Damit konnte der Wechsel der organisatorischen und finanziellen Zuständigkeit für heilpädagogische Maßnahmen von Kindern mit Behinderung vom Landeswohlfahrtsverband Hessen auf die kreisfreien Städte und Landkreise in Hessen erfolgen. Vertragspartner der bis zum heutigen Tag gültigen Rahmenvereinbarung sind die kommunalen Spitzenverbände sowie die Liga der Freien Wohlfahrtspflege in Hessen. In einer ergänzenden Vereinbarung hat das Hessische Sozialministerium seine vormals über eine Verwaltungsrichtlinie geregelte finanzielle Förderung rechtsverbindlich mit den genannten Vertragspartnern abgeschlossen.

\section{Integration braucht Personal}

Die Integration von Kindern mit Behinderung erfolgt in Hessen durch die Betreuung in einer regulären Kindertageseinrichtung des Stadtteils oder Ortes. Wenn möglich, sollte die Einrichtung wohnortnah, zumindest im sozialräumlichen Lebenskontext der Eltern und des Kindes gelegen sein. Darüber hinaus ist bei dem Leistungsangebot der Einrichtung den individuellen Anforderungen des Hilfebedarfs des Kindes sowie dem Wunsch und Wahlrecht der Eltern Rechnung zu tragen.

Wird ein Kind mit Behinderung in die Gruppe einer Einrichtung aufgenommen, führt dies prinzipiell zu einer Absenkung der Gesamtgruppenstärke von 25 Kindern (allgemeine Gruppenstärke nach der Verordnung über Mindestvoraussetzungen in Tageseinrichtungen für Kinder in Hessen) auf 20 Kinder. Damit wird der reguläre Betreuungsschlüssel Fachkraft/ Kind von 1,5 Fachkräften pro Kindergruppe zugunsten einer Verbesserung der Betreuungsintensität verändert. Mit einer 
weiteren Aufnahme von Kindern mit Behinderung in diese Gruppe, muss die Gesamtgruppenstärke weiterhin abgesenkt werden, so dass bei einer maximal möglichen Aufnahme von fünf Kindern mit Behinderung maximal zehn Kinder ohne Behinderung in der Gruppe betreut werden können.

Für die Sicherstellung der zusätzlichen Hilfen und Leistungen wird dem jeweiligen Träger, ausgehend vom bereits vorhandenen Personalbestand der Gruppe, zusätzliches Betreuungspersonal in Höhe von 15 Wochenstunden einer Fachkraft pro Kind mit Behinderung pauschal zur Verfügung gestellt. Es wird davon ausgegangen, dass im Rahmen dieser Zeitpauschale die im Einzelfall notwendigen Maßnahmen im Zusammenwirken mit den anderen Fachkräften, durch die reguläre Betreuungsarbeit umgesetzt werden. Mit der pauschalen Kontingentierung von 15 Wochenstunden pro Platz, die sich auf die gesamte Öffnungszeit der Tageseinrichtung bezieht, wird strukturell gewährleistet, dass sich der Betreuungsschlüssel kontinuierlich erhöht. Bei der Aufnahme von fünf Kindern mit Behinderung verfügt die Gruppe demnach über 3,5 Fachkräfte zur Betreuung aller Kinder.

\section{Integration braucht Konzepte}

Jede Kindertageseinrichtung ist mit dem Abschluss der Rahmenvereinbarung Integrationsplatz vor die Aufgabe gestellt, Kindern mit Behinderung einen Platz in ihrer Einrichtung zur Verfügung zu stellen.

Die Rahmenvereinbarung verpflichtet die Kindertageseinrichtungen nicht explizit zur Anpassung des pädagogischen Konzepts hinsichtlich der Anforderung der Integrationsleistungen, zeigt jedoch, dass die vorhandenen Struktur und Prozessqualitäten daran auszurichten sind. So muss das Raumprogramm die erforderlichen pädagogischen Differenzierungen sowohl innerhalb der Gruppe als auch gruppenübergreifend gewährleisten. Darüber hinaus muss die medizinischtherapeutische Förderung des Kindes möglich sein. Wenn mehr als drei Kinder mit Behinderung in der Gruppe betreut werden, muss innerhalb der vorhandenen Räumlichkeiten ein geeigneter Mehrzweckbereich, sowie ein für die Einzelför- derung geeigneter Raum vorhanden sein. Die Prüfung der Eignung ist an das Betrieberlaubnisverfahren nach $\mathbb{S} 45 \mathrm{SGB}$ VIII angebunden und wird durch das örtliche Jugendamt und das Hessische Sozialministerium verbindlich durchgeführt.

Die Träger sind nach der Rahmenvereinbarung prinzipiell angehalten, sowohl Aktivitäten und Maßnahmen zur Qualitätsentwicklung für die eigene Kindertageseinrichtung, als auch in ihrem regionalen Umfeld mit anderen sozialen Institutionen (Schule, Frühförderung etc.) zu entwickeln. Qualitätsentwicklungsmaßnahmen sind beispielsweise die Entwikklung und Fortschreibung des Konzeptes, der Aufbau eines Qualitätszirkels sowie die Gründung einrichtungsübergreifender themenbezogener Arbeitskreise.

Mit der Aufnahme eines Kindes mit Behinderung ist der Träger verpflichtet, seinen Fachkräften die Gelegenheit zu geben, an heil und behindertenpädagogischen Fortbildungsveranstaltungen teilzunehmen, sowie praxisbegleitende Beratungsangebote, insbesondere die der Fachberatung zu nutzen.

Die Finanzierung des Integrationsplatzes erfolgt durch den örtlichen Sozialhilfeträger mittels einer jährlich Maßnahmenpauschale in Höhe von 16.595 Euro pro Platz, sowie durch eine ergänzende, ebenfalls jährliche Integrationspauschale in Höhe von 1.500 Euro pro Platz durch das Hessische Sozialministerium im Rahmen des Hessischen Kinder und Jugendhilfegesetzes.

\section{Resümee}

Die hessische »Rahmenvereinbarung Integrationsplatz « hat in ihrer fast zehnjährigen Laufzeit zu dem fulminanten Anstieg um rund eintausend auf 4.589 Integrationsplätze (2006) in Kindertageseinrichtungen geführt. Die Eltern haben also das Angebot einer wohnortnahen und stärker auf Normalisierung und Inklusion konzeptionierten Betreuung der Kinder sehr gut angenommen haben. Es ist anzunehmen, dass die Steigerungsrate auf eine hohe Akzeptanz der Angebote zurückgeführt werden kann, da die Leistungen nun sicherlich auch von Eltern in Anspruch genommen wurden, die bisher ihre Kinder eigenständig zu Hause oder nicht im Rahmen von teilstationären Angeboten betreuten. Auf Grund der hohen
Nachfrage wurde jedoch das primäre Ziel der Kostenträger auf Reduzierung der administrativen Aufwendungen und Einsparungen der finanziellen Mittel nicht erreicht.

Das veränderte Verständnis von Integration in den bestehenden Regeleinrichtungen hat positiv zu einer Veränderung des Institutionenbegriffs mit längst notwendigen Veränderungen von Arbeitsstrukturen, Verfahren und Routinen beigetragen. Zugleich hat es zu einer Fortentwicklung des Fachpersonals in Kindertageseinrichtungen hin zu einem gemeinsamen Bewusstsein der Umsetzung von integrativer Erziehung und Bildung geführt.

Eine Aufgabe für die Zukunft ist es, von der gegenwärtigen Integrationspraxis von Kindern mit Behinderung zur Praxis der umfassenden Inklusion zu gelangen. Dabei geht es nicht mehr nur um die Eingliederung in die Regelkindertageseinrichtung, die Diagnose und Begutachtung der Defizite durch Experten und eine in der Gruppenrealität angewandte ZweiGruppenTheorie der Betreuungs und Förderkonzepte. Erforderlich ist vielmehr auch, das gemeinsame Leben und Lernen von Kindern mit und ohne Behinderung im Rahmen des Erziehungsauftrages des SGB VIII zu ermöglichen, um eine sozialdiagnostisch unterstützte Problemlösungskompetenz eines ErzieherinnenTeams sowie um die personelle und konzeptionelle Bereitstellung entsprechender Ressourcen für ein gemeinsames System von Kinderbetreuung.

Erforderlich ist also: eine Verbesserung der strukturellen Rahmenbedingungen der einzelnen Regeleinrichtungen selbst, eine veränderte Ausbildungs und Fortbildungspraxis des Fachpersonals und eine weitere Zusammenführung der unterschiedlichen Rechtsbezüge. Längst überfällig ist zudem eine Ausweitung der Integrationsleistungen für Kinder unter drei Jahren und für Grundschulkinder. 